\title{
Evaluación, aplicación, difusión y abastecimiento de un sistema de generación eólica
}

Evaluation, implementation, distribution and supply of wind power

generation system

Recibido: julio 10 de 2015 | Revisado: agosto 21 de 2015 | Aceptado: octubre 02 de 2015

\author{
Ana Quispe ${ }^{1}$ \\ Jorge CALDerón
}

\begin{abstract}
This research was initiated due to the low diffusion of the use of wind power is our country and aims to be an example in order to increase the use and dissemination of this type of renewable energy. It's mainly an assessment of the use and application of a wind power generation system, which will have a power supply that enables use over an untimely power cut. The analysis of the actual data obtained from readings of airspeed allowed us to establish how to use the energy from the wind powered generator, determining that it has a major influence in the place of the location of the generator, the temperature and the time required to obtain the renewable energy.
\end{abstract}

Keywords: wind power, generator, renewable energy

\section{Resumen}

Esta investigación se inició debido a la baja difusión de la utilización de la energía eólica en nuestro país y pretende ser un ejemplo para aumentar el uso y la difusión de este tipo de energía renovable. Es básicamente una evaluación de la utilización y aplicación de un sistema de generación de energía eólica, que contará con una fuente de alimentación que permita su utilización durante un corte de energía a destiempo. El análisis de los datos reales obtenidos de las lecturas de velocidad del aire, nos permitió establecer cómo utilizar la energía del generador de energía eólica, determinando que ejerce mucha influencia en el lugar de la ubicación del generador, la temperatura y el tiempo de obtención de la energía renovable.

Palabras clave: energía eólica, generador, energía renovable

1 Facultad de Ingeniería y Arquitectura. de la Universidad de San Martín de Porres.

aquisper@usmp.pe 


\section{Introducción}

La presente investigación es una contribución a la búsqueda de fuentes de energías renovables, ya que nuestra matriz energética está por lo general basada, fundamentalmente, en hidrocarburos y es la energía eólica una fuente de energía renovable que ofrece a las generaciones venideras, una esperanza de un futuro en el que el mundo no dependa de la energía no renovable, la cual genera mucha contaminación.

Nuestra preocupación nace como una solución a una situación que ha llamado la atención y es el alto grado de consumo de energía eléctrica en las instalaciones de la Facultad de Ingeniería y Arquitectura de la Universidad de San Martín de Porres.

Asimismo, somos partícipes del uso de energías que apoyen al medio ambiente, que no generen residuos peligrosos como el dióxido de carbono y que no produzcan otros contaminantes asociados con la generación de energía a partir de combustibles fósiles.

Los cambios climáticos que se están presentando en el mundo actual han originado que el uso de la energía eólica vaya cada vez más en aumento; por ello, se requieren utilizar formas de obtención de energía que no afecten el ambiente. El Perú no es ajeno a estos cambios y por eso el Estado Peruano ha decidido impulsar la aplicación de las energías renovables.

El buen uso de los recursos naturales, como manera de combatir el cambio climático global, ha motivado, en gran medida, a que realicemos esta investigación, de manera que se pueda utilizar un generador eólico que mantenga el alumbrado en las aulas y/o laboratorios de la Facultad de Ingeniería y Arquitectura de la Universidad de San Martín de Porres.

Es importante tomar en cuenta que el generador eólico no genera ruidos molestos en la ingeniería moderna. Los ruidos se han re- ducido a gran escala, esto es ventajoso puesto que no generará incomodidad entre el personal docente, administrativo y alumnos.

\section{Energía eólica}

La energía eólica es aquella que procede del movimiento del aire o viento. El viento puede ser de diferente velocidad, en diferentes puntos de la Tierra y también se ve afectada por la densidad del aire.

La velocidad del viento está cambiando continuamente, por tanto, es deseable describirlo de acuerdo con los modelos probabilísticos. La densidad de probabilidad función (pdf) de la velocidad del viento es importante en numerosas aplicaciones de la energía eólica (Nemes y Munteanu, 2010).

\subsection{Ventajas y desventajas de la ener- gía eólica}

Entre las ventajas, podemos nombrar que la energía eólica es renovable porque proviene de fuentes naturales inagotables, no contamina, $y$ es capaz de regenerarse por medios naturales.

Asimismo, podemos decir que las instalaciones eólicas tienen un pequeño impacto ambiental y son fuentes que pueden competir con otras fuentes energéticas tradicionales como las centrales térmicas de carbón.

Es importante tener en cuenta que la energía eólica es fácil de integrar en redes de potencia eléctrica ya existentes.

Como desventaja se puede mencionar, que en ocasiones, la velocidad del viento es muy baja y no logra cubrir las necesidades de energía que se requiere. Si se quiere lograr mayor 
energía se tiene que recurrir al uso de equipos eólicos más grandes, que son más costosos y muchas veces no se cuenta con espacio ni instalaciones para colocarlos.

En diversos lugares, el viento adquiere diversas velocidades y esto se debe a diversos factores como la temperatura y la brisa de mar que afectan su origen, por lo que si se quiere ubicar un equipo eólico, en un distrito, en el que no haya mucho viento, este no será de gran utilidad, porque la energía no cubrirá las necesidades específicas.

\subsection{Uso del viento como energía}

Según la Asociación Mundial de Energía Eólica (WWEA, 2013), el uso de la energía eólica ha ido en un aumento cada vez mayor y esto se puede demostrar, en el último reporte publicado en su sitio Web, donde precisan que la capacidad eólica en todo el mundo alcanzó 296'255 MW a finales de junio de 2013, de los cuales 13 '980 MW se incrementaron en los últimos seis meses del año. Este aumento es menor que el que se registró a mediados de los años 2011 y 2012.

Total Installed Capacity 2010-2013 [MW]

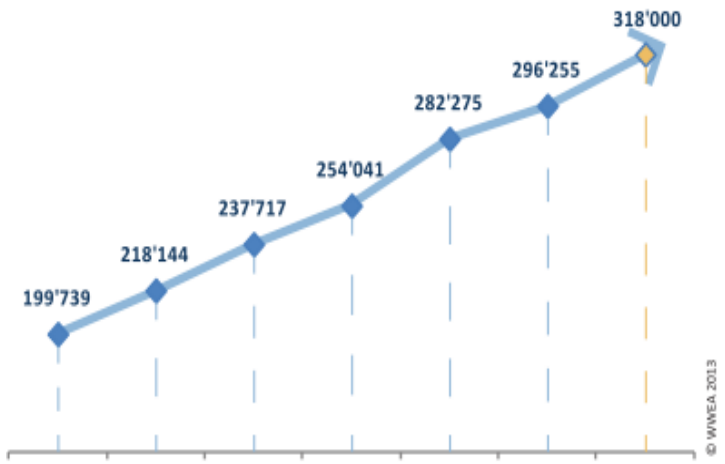

End 2010 Mid 2011 End 2011 Mid 2012 End 2012 Mid 2013 End 2013*

Figura 1. Capacidad eólica en todo el mundo World Wind Energy Association (2013).
La utilización de la energía eólica para la generación de electricidad desempeñará un papel importante en el suministro de electricidad en muchos países del futuro. En Alemania, actualmente, representa aproximadamente el 6\% del suministro de electricidad. Su participación se prevé que aumente alrededor del 15\% en el año de 2020 (Sanz-Casado, García-Zorita, Eleazar Larsen e Ingwersen, 2007).

\subsection{Naturaleza del viento}

El viento está presente en la superficie de la Tierra, conocido como el movimiento de los gases que rodean un planeta o cuerpo astronómico. Alrededor del 1 al 2\% de la energía que proviene del sol se convierte en energía eólica.

A escala global, se debe a la diferencia de temperatura que provoca la energía solar sobre las distintas partes del globo terrestre. Las diferencias de densidad y presión originadas por estas variaciones de temperatura son las causas del movimiento de las masas de aire (Villarrubia, 2012).

Los vientos se originan a causa de la presión atmosférica y las diferencias se producen debido a las diferentes temperaturas que tenemos en el planeta. El aire frío tiende a desplazarse hacia abajo, mientras que el aire caliente se desplaza hacia arriba.

Desde años anteriores, en la agricultura, se utilizaban molinos de viento para moler granos y bombear el agua del subsuelo y lo marineros utilizaban velas para capturar el viento y realizar sus viajes. En los últimos años, el uso de las turbinas eólicas para generar energía ha ido en aumento. Pero eso aún es poco ya que constituyen una pequeńa 
fracción de toda la energía utilizada en el mundo.

\subsection{Potencia eólica}

La energía del viento se puede usar para generar la potencia eólica; de esta manera, un sistema de energía del viento transforma la energía del viento en energía mecánica o eléctrica.

Todas las turbinas eólicas instaladas, alrededor del mundo, a mediados del ańo 2013 pueden generar alrededor del 3,5\% de la demanda de electricidad de la población mundial.

La capacidad eólica mundial creció un $5 \%$ en un plazo de seis meses (después de 7\% en el mismo periodo de 2012 y el $9 \%$ en 2011) y un $16,6 \%$ sobre una base anual (a mediados de 2013 en comparación con el año 2012). En comparación, la anual tasa de crecimiento en el año de 2012 fue significativamente mayor (19\%) (Sanz-Casado et al., 2007).

La energía contenida en el viento es energía cinética. La potencia que transporta una corriente de aire se puede calcular recordando que se define como la energía utilizada o convertida por unidad de tiempo (Villerrubia, 2012), de la siguiente manera (Figura 2):

$$
\rho=\mathrm{E}_{\mathrm{c}} / \tau=\left[(1 / 2) \mathrm{m}_{\mathrm{u}_{0}}^{2}\right] / \tau=\left[(1 / 2)\left(\rho\left(\begin{array}{ll}
1 & 2 \\
\mathrm{u}_{0}
\end{array}\right] / \tau=(1 / 2) \rho 1 / 2\right) \tau\right) \mathrm{u}_{0}^{2}=(1 / 2) \rho 1 \quad \begin{aligned}
& 3 \\
& \mathrm{u}_{0}
\end{aligned}
$$

Donde:

$\rho=$ densidad del aire $=1,2256 \mathrm{kgm}^{-\mathrm{J}} ; \mathrm{m}=$ masa $=\rho \mathrm{V} ; \mathrm{V}=\mathrm{A}$

$\mathrm{A}=$ área barrida; $1 / \tau=$ longitud recorrida en la unidad de tiempo $=$

$\mathrm{u} 0, \mathrm{y} \mathrm{u} 0=$ velocidad no perturbada del viento.

Figura 2. Fórmula de potencia que transporta una corriente de aire

\subsection{Variabilidad de la velocidad el viento}

La velocidad del viento es muy variable, esto se comprueba en las mediciones efectuadas en el análisis de esta investigación, ya que el contenido energético del viento varía continuamente. Por tanto, la turbina eólica limita la producción de energía conforme varíe el viento.

En nuestro planeta, el viento sopla más fuerte durante el día que en la noche. Estas variaciones se deben a las diferencias de temperatura entre la superficie del mar y la terrestre. El viento presenta turbulencias y tiende a cambiar de dirección rápidamente durante el día, dado que la mayor parte de la energía eólica se produce en horario diurno y ello es favorable.

El conocimiento de las propiedades estadísticas de la velocidad del viento es esencial para la predicción de la salida de energía de un sistema de conversión de energía eólica (Sanz-Casado, García, Eleazar, Larzen \& Inqwersen, 2013).

Debido a que el viento varía en diferentes horas y dependiendo del lugar, es relevante medir y analizar la energía y con esto establecer en qué momento se obtendrá mayor energía y en qué lugar sería más conveniente colocar el equipo eólico. 


\subsection{Equipos de medición de velocidad del viento}

Por lo tanto, la turbina eólica tendrá limitada la producción de energía conforme varíe el viento.

Los instrumentos de medición de la velocidad del viento se conocen como anemómetros. La dirección del viento se mide ya sea usando un equipo de medición para condiciones climatológicas (menguante de tiempo) o por medio del anemómetro mismo (Nemes y Munteanu, 2011).

Existen los anemómetros de rotación, de tubo de Pilot, de hilo caliente, de medición por ultrasonido, láser, o efecto Doppler (SODAR).

Los anemómetros rotatorios son, por lo general, para uso industrial y comercial, miden la velocidad, el volumen y temperatura del aire. Son los más utilizados debido a su facilidad de uso y obtención de resultados. Actualmente, existen modelos digitales que facilitan ver los resultados.

Los anemómetros de rotación son de tipo cazoleta o de hélice. Debido a la simplicidad y suficiente exactitud, los anemómetros de hélice se utilizan con mayor regularidad; asimismo, permiten hacer una medición a distancia.

Para poder seleccionar el anemómetro adecuado para hacer las mediciones de viento debemos tener presente lo siguiente:

a. Alineación entre la velocidad del viento y la señal de salida.

b. La medida de la velocidad no debe ser afectada por las variaciones de densidad, temperatura, presión y humedad del aire.

$$
\begin{aligned}
& \text { c. Calibración y precisión del } \\
& \text { equipo. }
\end{aligned}
$$

\section{Uso de la energía eólica}

El uso de la energía del viento o energía eólica data de muchos años atrás en que fue utilizada comúnmente en procesos mecánicos. La energía procedía a través de grandes molinos de viento, luego era transformada y entregada a través del eje de la turbina. Esta energía obtenida fue utilizada en procesos mecánicos como bombear agua, moler granos y en los últimos años, para la generación de la electricidad.

El uso de la energía eólica cada vez está tomando mayor importancia en el mundo y en las investigaciones. Este tema está en crecimiento y se debe a que esta energía no afecta al medio ambiente, no causa dańos, desastres ecológicos. Tampoco contribuye al agrandamiento de la capa de ozono, al calentamiento global ni emite gases tóxicos.

En el año 2011, la energía eólica representó el 30\% de la nueva capacidad de energía renovable en la Unión Europea con las nuevas instalaciones de energía solar que constituyen la mayor proporción $(66 \%)$. En la mezcla de capacidad de energía total de la Unión Europea, la cuota de energía eólica es del 10\% y la Energía Solar del 5\% (Al-Shemmeri, 2010).

\section{Instalaciones eólicas}

\subsection{Tipos de generadores eólicos}

Existen dos tipos de generadores eólicos: de eje horizontal y de eje vertical (Qian y He, 2011).

En la Figura 3, podemos ver la clasificación de los generadores. Los de eje horizontal son los más utilizados y de mayor potencia. Utili- 
zan las hélices para atrapar el viento a través de las hojas de rotación y luego impulsan el generador eléctrico. Los modelos más grandes se encuentran en los parques eólicos.

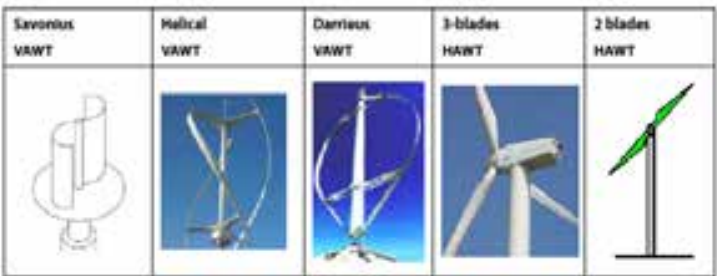

Figura 3. Tipos de turbinas de viento (Qian y He, 2011).

La turbina de poder de viento vertical no necesita estar frente a la dirección del aire cuando esta cambie. Esto es una gran ventaja en comparación con los ejes de la turbina eólica horizontal. Esto no solo simplifica el diseño de la estructura, sino que también reduce la fuerza de la tapa superior cuando la rueda de viento esté frente a la dirección del viento (Silva y Montoya, 2008).

Uno de los principales problemas con la tecnología de eje horizontal es que tiene problemas para aprovechar las velocidades más bajas, en comparación con las nuevas turbinas eólicas de eje vertical y de gran altitud.

\section{Materiales y método}

\subsection{Componentes de un generador eólico}

La turbina de poder de viento vertical no necesita estar frente a la dirección del aire cuando esta cambie. Esto es una gran ventaja en comparación con los ejes de la turbina eólica horizontal. Esto no solo simplifica el diseño de la estructura, sino que también reduce la fuerza de la tapa superior cuando la rueda de viento esté frente a la dirección del viento (Silva y Montoya, 2008).
Uno de los principales problemas con la tecnología de eje horizontal es que tiene problemas para aprovechar las velocidades más bajas, en comparación con las nuevas turbinas eólicas de eje vertical y de gran altitud.

Por lo general, los elementos principales de un generador eólico son:

- El rotor

- La caja de engranajes

- Un generador

- El sistema de control y protección

- La torre

- La base

En la Figura 4, podemos ver la estructura de un molino eólico, una construcción que puede llegar a medir hasta 200 metros de altitud.

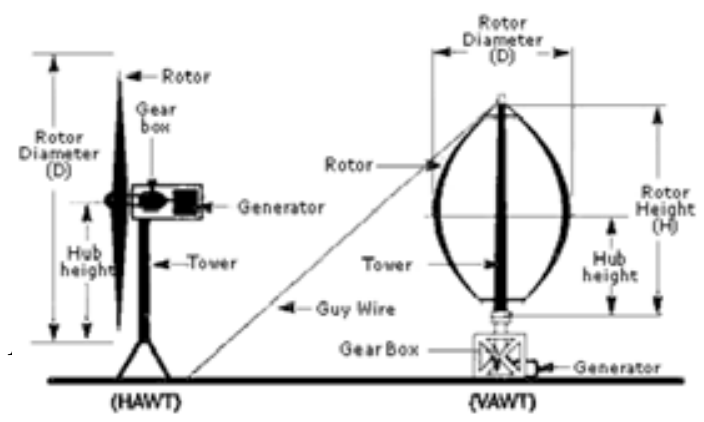

\subsection{Diseño de generadores eólicos}

En el diseño de generadores eólicos, tenemos que tener en cuenta las siguientes consideraciones:

- Como los generadores están sujetos a fuerzas fluctuantes, se deben tener consideraciones básicas de carga.

- Los tipos de generadores que se van a utilizar, son máquinas de eje horizontal y vertical.

- Utilización de generadores con rotor barlovento (rotor con cara al viento) o sotavento (rotor si- 
tuado en la cara a sotavento de la torre).

- Las palas que debe tener el generador eólico, por lo general, tienen diseños tripala, con el rotor barlovento.

- Tener en cuenta un bajo ruido mecánico y aerodinámico en generadores eólicos.

\section{Evaluaciones de uso de un generador eólico}

\subsection{Estudio de la velocidad del aire}

Con el fin de implementar un sistema de energía eólica se tomaron algunas muestras de la velocidad del aire en el distrito de La Molina, lugar donde se encuentra ubicada la Facultad de Ingeniería y Arquitectura. Los resultados se pueden observar en la Tabla 1.

Tabla 1

Estudio de la velocidad del aire (velocidad y temperatura)

\begin{tabular}{|c|c|c|c|c|c|c|c|c|c|c|c|}
\hline Pab. & Fecha & Hora & $\begin{array}{c}\text { Velocidad } \\
\mathrm{m} / \mathrm{s}\end{array}$ & $\begin{array}{c}\text { Velocidad } \\
\mathrm{Km} / \mathrm{h}\end{array}$ & $\begin{array}{c}\text { Tempe- } \\
\text { ratura }\end{array}$ & Pab. & Fecha & Hora & $\begin{array}{c}\text { Velocidad } \\
\mathrm{m} / \mathrm{s}\end{array}$ & $\begin{array}{c}\text { Velocidad } \\
\mathrm{Km} / \mathrm{h}\end{array}$ & $\begin{array}{c}\text { Tempe- } \\
\text { ratura }\end{array}$ \\
\hline \multirow{20}{*}{ 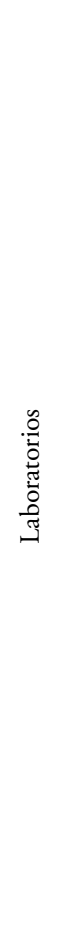 } & $14 / 02 / 2014$ & $16: 00$ & 4.5 & 12.5 & 27.3 & \multirow{20}{*}{ 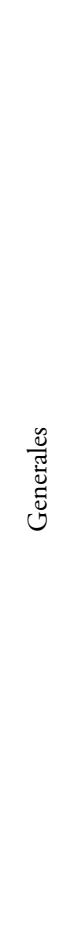 } & $24 / 02 / 2014$ & $16: 00$ & 1.2 & 3.33 & 25.1 \\
\hline & $14 / 02 / 2014$ & $18: 00$ & 2.5 & 6.94 & 22.5 & & $24 / 02 / 2014$ & 18:00 & 3.5 & 9.72 & 25 \\
\hline & $14 / 02 / 2014$ & $20: 00$ & 1.5 & 4.17 & 22.3 & & $24 / 02 / 2014$ & 20:00 & 4.2 & 11.67 & 24.1 \\
\hline & $14 / 02 / 2014$ & $22: 00$ & 1.5 & 4.17 & 22 & & $24 / 02 / 2014$ & 22:00 & 4.4 & 12.22 & 23.4 \\
\hline & $17 / 02 / 2014$ & $16: 00$ & 4 & 11.11 & 27.6 & & $25 / 02 / 2014$ & 10:00 & 2.6 & 7.22 & 28.4 \\
\hline & $17 / 02 / 2014$ & 18:00 & 2.6 & 7.22 & 24.2 & & $25 / 02 / 2014$ & $12: 00$ & 2.4 & 6.67 & 28.8 \\
\hline & $17 / 02 / 2014$ & $20: 00$ & 1.4 & 3.89 & 22 & & $25 / 02 / 2014$ & $14: 00$ & 3.8 & 10.56 & 28.1 \\
\hline & $17 / 02 / 2014$ & 22:00 & 1.3 & 3.61 & 21 & & $25 / 02 / 2014$ & 16:00 & 3.2 & 8.89 & 27.8 \\
\hline & $18 / 02 / 2014$ & $10: 00$ & 3.4 & 9.44 & 28.2 & & $26 / 02 / 2014$ & 16:00 & 3.8 & 10.56 & 26.2 \\
\hline & $18 / 02 / 2014$ & $12: 00$ & 4.6 & 12.78 & 28 & & $26 / 02 / 2014$ & 18:00 & 4.2 & 11.67 & 26.8 \\
\hline & $18 / 02 / 2014$ & 14:00 & 4.2 & 11.67 & 28 & & $26 / 02 / 2014$ & 20:00 & 3.6 & 10 & 25.2 \\
\hline & $18 / 02 / 2014$ & $16: 00$ & 3.8 & 10.56 & 27.8 & & $26 / 02 / 2014$ & $22: 00$ & 4.8 & 13.33 & 25 \\
\hline & $19 / 02 / 2014$ & $16: 00$ & 2.4 & 6.67 & 28 & & $27 / 02 / 2014$ & 10:00 & 2.5 & 6.94 & 28.3 \\
\hline & $19 / 02 / 2014$ & 18:00 & 1.5 & 4.17 & 24 & & $27 / 02 / 2014$ & $12: 00$ & 3.8 & 10.56 & 28.1 \\
\hline & $19 / 02 / 2014$ & 20:00 & 2.2 & 6.11 & 23.5 & & $27 / 02 / 2014$ & $14: 00$ & 4.5 & 12.5 & 27.8 \\
\hline & $19 / 02 / 2014$ & $22: 00$ & 0.5 & 1.39 & 21.2 & & $27 / 02 / 2014$ & $16: 00$ & 4.8 & 13.33 & 27.7 \\
\hline & $20 / 02 / 2014$ & 10:00 & 4.2 & 11.67 & 28 & & $28 / 02 / 2014$ & $16: 00$ & 3.1 & 8.61 & 26.3 \\
\hline & $20 / 02 / 2014$ & $12: 00$ & 3.4 & 9.44 & 27.6 & & $28 / 02 / 2014$ & 18:00 & 4.3 & 11.94 & 26.5 \\
\hline & $20 / 02 / 2014$ & 14:00 & 2.8 & 7.78 & 28 & & $28 / 02 / 2014$ & 20:00 & 4 & 11.11 & 26.1 \\
\hline & $20 / 02 / 2014$ & $16: 00$ & 3.3 & 9.17 & 27.3 & & $28 / 02 / 2014$ & $22: 00$ & 4.6 & 12.78 & 26 \\
\hline
\end{tabular}

Para hacer las pruebas de la velocidad del aire cuyos resultados se encuentran en la Tabla 1, se utilizó un anemómetro digital de rotación tipo hélice marca Benetech que fue adquirido por la Facultad de Ingeniería y Arquitectura debido a que entre los diversos modelos que hay en el mercado, era el que mejor se acoplaba a las necesidades de la investigación.

\section{2 Áreas donde hay mayor influen- cia del viento en Lima}

En el estudio realizado por el Servicio Nacional de Meteorología e Hidrología del Perú (SENAMHI) en el año 2008, vemos que la información horaria de viento superficial (velocidad y dirección) correspondiente al mes de agosto es de $15 \mathrm{~m} / \mathrm{s}$ para el período matutino $(07: 00-11: 00)$, vespertino $(12: 00$ 
- 18:00) y nocturno (19:00 - 06:00), respectivamente. Este análisis es importante por cuanto esta variable meteorológica influye directamente en los procesos de transporte y dispersión de los contaminantes determinando tanto la localización como los niveles altos o bajos de concentración de estos.

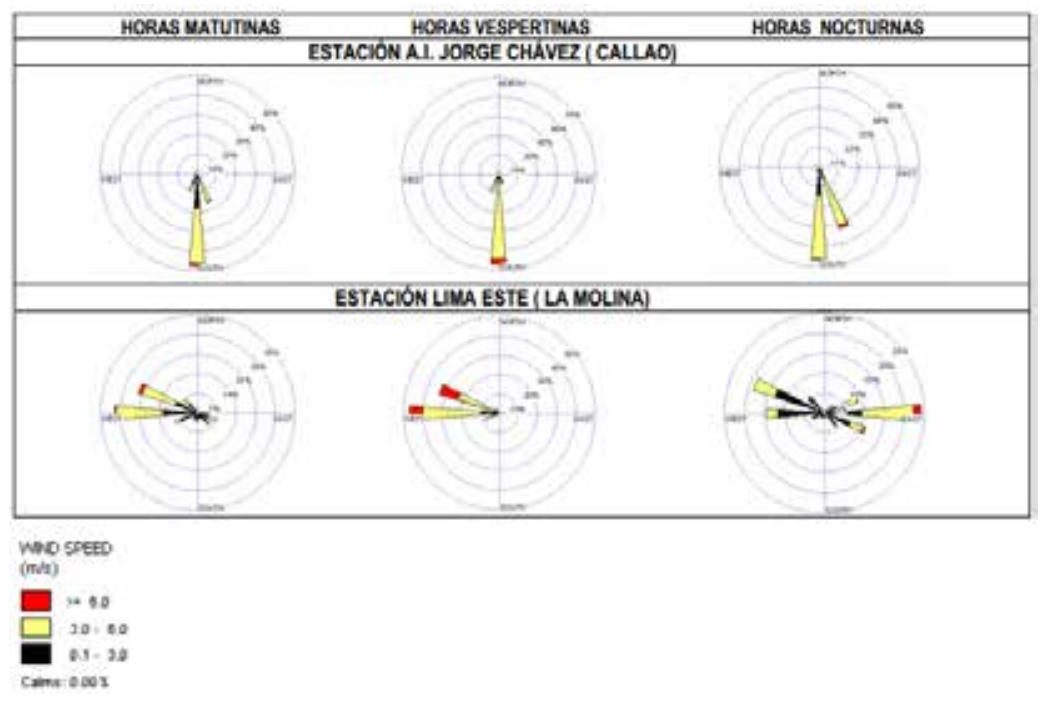

Figura 4. Rosas de viento (m/s) en las estaciones de El Callao y La Molina. Silva y Montoya (2008).

Asimismo, podemos ver que durante el período matutino (7:00 a 11:00 horas), predominaron vientos de intensidad media débil $(<3 \mathrm{~m} / \mathrm{s})$ en ambas estaciones analizadas, con intensidades comprendidas entre 2,3 a $2,7 \mathrm{~m} / \mathrm{s}$. Se registró ocurrencia de calmas en la Estación Callao (20,7 \%).

Durante el período vespertino (12:00 a 18:00 horas) se registraron vientos de intensidades moderadas en el Callao
$(3,8 \mathrm{~m} / \mathrm{s})$ y La Molina $(4,3 \mathrm{~m} / \mathrm{s})$. No se registró ocurrencia significativa de calmas. Durante el período nocturno (19:00 a 6:00 horas) se registraron vientos de intensidad débil en el Callao y La Molina con registros. No se registró ocurrencia significativa de calmas.

En la Tabla 2, se puede apreciar el resumen de la velocidad y dirección del viento en el año 2008 durante el día y la noche.

\section{Tabla 2}

Información horaria de viento superficial (velocidad y dirección)

\begin{tabular}{|l|l|l|l|}
\hline Distrito & Día & Noche & Hora \\
\hline La Molina & $13 \mathrm{Km} / \mathrm{h}$ & $8 \mathrm{Km} / \mathrm{h}$ & $20: 27$ \\
\hline Jesús María & $11 \mathrm{Km} / \mathrm{h}$ & $11 \mathrm{Km} / \mathrm{h}$ & $20: 27$ \\
\hline Surco & $55 \mathrm{Km} / \mathrm{h}$ & $13 \mathrm{Km} / \mathrm{h}$ & $20: 28$ \\
\hline Lima & $11 \mathrm{Km} / \mathrm{h}$ & $10 \mathrm{Km} / \mathrm{h}$ & $20: 29$ \\
\hline Ancón & $13 \mathrm{Km} / \mathrm{h}$ & $8 \mathrm{Km} / \mathrm{h}$ & $20: 29$ \\
\hline Barranco & $11 \mathrm{Km} / \mathrm{h}$ & $11 \mathrm{Km} / \mathrm{h}$ & $20: 30$ \\
\hline Miraflores & $45 \mathrm{Km} / \mathrm{h}$ & $2 \mathrm{Km} / \mathrm{h}$ & $20: 31$ \\
\hline San Isidro & $11 \mathrm{Km} / \mathrm{h}$ & $8 \mathrm{Km} / \mathrm{h}$ & $20: 33$ \\
\hline Pueblo Libre & $11 \mathrm{Km} / \mathrm{h}$ & $10 \mathrm{Km} / \mathrm{h}$ & $20: 34$ \\
\hline San Miguel & $14 \mathrm{Km} / \mathrm{h}$ & $6 \mathrm{Km} / \mathrm{h}$ & $20: 39$ \\
\hline Ate & $13 \mathrm{Km} / \mathrm{h}$ & $8 \mathrm{Km} / \mathrm{h}$ & $20: 40$ \\
\hline
\end{tabular}

Nota: Huler, 2004. 


\subsection{Efectos de la temperatura sobre la velocidad del viento}

El viento es el aire en movimiento. Es un factor importante del ciclo hidrológico porque influye en el transporte del calor y de la humedad en el proceso de evaporación.
La velocidad se expresa en $\mathrm{m} / \mathrm{sg}, \mathrm{Km} / \mathrm{h}$ o en nudos $(1$ nudo $=0.514 \mathrm{~m} / \mathrm{sg}=$ $1.85 \mathrm{Km} / \mathrm{h})$.

La escala de Beaufort que consta de 13 grados nos muestra el orden de magnitud de la velocidad de los vientos (San Martín, Zamora, Apenibay y Egvia, 2010).

Tabla 3

Escala de Beaufort

\begin{tabular}{|l|l|}
\hline Calma $\mathrm{O}-1 \mathrm{Km} / \mathrm{h}$. & Viento muy fuerte $45-54$. \\
\hline Ventolina $2-6$. & Temporal $55,-65$ \\
\hline Viento suave $7-12$. & Temporal fuerte $66-77$ \\
\hline Viento leve $13-18$. & Temporal muy fuerte $78-90$ \\
\hline Viento moderado $19-26$. & Tempestad $91-104$ \\
\hline Viento regular $27-35$. & Huracán $>104$ \\
\hline Viento fuerte $36-44$. & \\
\hline
\end{tabular}

Nota: Extraído de San Martín et al. (2010).

Existe un fenómeno denominado "Inversión de temperatura" que ocurre cuando la temperatura aumenta o baja dependiendo de ciertas condiciones. Por ejemplo, en las primeras horas del día, la Tierra se encuentra a baja temperatura debido a que en la noche ha perdido gran cantidad de calor; en ausencia de vientos y con el cielo despejado, las capas inferiores de la troposfera son más frías que las inmediatas superiores como consecuencia la temperatura sube con la altura.

Esta inversión de temperatura tiende a ser destruida por la mezcla que producen los vientos fuertes próximos al suelo y desde luego, el calentamiento que sigue a la salida del sol termina por restablecer el gradiente normal de temperatura.

Asimismo, durante el día o la noche, la temperatura va cambiando debido al efecto de la brisa de mar.

\section{Uso de la energía del generador eólico}

\subsection{Cantidad de energía almacenada de un generador}

Entre los muchos usos del procesamiento digital de imágenes, espe- cíficamente, en esta investigación nos centramos en el apoyo durante el control calidad y selección de los alimentos para aquellas personas que sufren algún tipo de deficiencia de visualización de colores, conocidos como daltónicos, quienes ven los colores de distinta manera a como lo ven la mayoría de personas.

La energía almacenada de un generador eólico se realiza en un banco de baterías, existen otros tipos de almacenamiento que se han desarrollado en los últimos años, como por ejemplo en la ciudad de Zhangbei en China, se tiene una alta capacidad de almacenamiento que llega hasta los $36 \mathrm{MWh}$.

En la Facultad de Ingeniería y Arquitectura se puede aprovechar el banco de baterías para el almacenamiento de energía proveniente del generador eólico.

Las tecnologías de almacenamiento utilizadas presentan criterios técnicos y económicos, que varían en función 
de las necesidades y aplicaciones específicas (Ibrahim, Ilinca y Person, 2008).

Las tecnologías de almacenamiento se pueden dividir en función de las aplicaciones (Enríquez, 2009):

- Aplicaciones de baja potencia en áreas aisladas.

- Aplicaciones de media potencia en áreas aisladas.

- Aplicaciones de conexión de red con la nivelación de pico aplicables para un sistema a gran escala.

- Aplicaciones de control de calidad de energía aplicables para un sistema a gran escala.

Específicamente, en el caso de la Facultad de Ingeniería y Arquitectura se propone el manejo de las aplicaciones de baja potencia, en áreas aisladas.

\subsection{Uso de la energía del generador}

Esta investigación se desarrolla con el fin de encontrar una solución alternativa para apoyar reducir los altos costos de energía eléctrica que se tienen actualmente en la Facultad de Ingeniería y Arquitectura, que ascienden a más de S/. 5000.00 nuevos soles al mes. En una primera instancia, se pensó en la utilización de un generador eólico para proveer de energía a los servidores que se encuentran en el centro de cómputo de la Universidad, pero en razón de los resultados de las pruebas desarrolladas en esta investigación, se determinó un mejor uso de la energía obtenida.

Además de las altas facturas de electricidad, el enorme consumo de energía de centros de datos también puede conducir a consecuencias ambientales negativas (Por ejemplo, las emisiones de $\mathrm{CO} 2$ y el calentamiento global), debido a sus grandes emisiones de carbono (Ibrahim et al., 2008).

En esta investigación, se realizó una revisión del sistema UPS (Uninterruptible Power Supply) con que cuenta la Facultad de Ingeniería y Arquitectura, que es un sistema de alimentación ininterrumpida, que está compuesto de baterías y otros elementos almacenadores de energía, que pueden proporcionar energía eléctrica por un tiempo limitado.

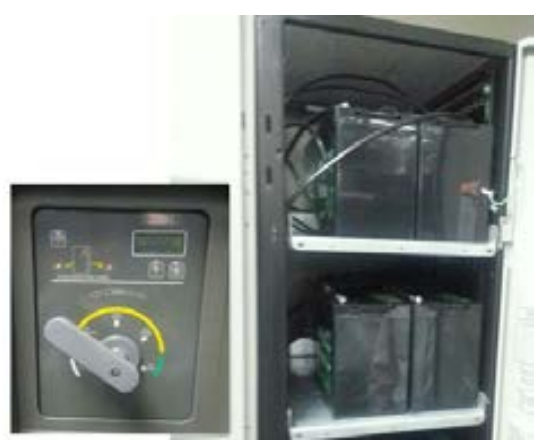

Figura 5. UPS del centro de cómputo de la Facultad de Ingeniería y Arquitectura.

Específicamente, en la Facultad de Ingeniería y Arquitectura, el UPS es utilizado cuando hay cortes de energía eléctrica para que los servidores, que se encuentran en el centro de cómputo, sigan funcionando con la energía que el UPS tiene almacenada que es de 35KVA.

Después de las pruebas que se hicieron, en esta investigación, y debido al requerimiento de energía de los servidores del centro de cómputo de la Universidad, se decidió proponer como utilización de la energía almacenada en la batería del generador eólico, a un grupo de luces que sean importantes para mantenerlas encendidas durante un corte de energía eléctrica. 
La máxima potencia que le podríamos extraer al viento, a través de un generador eólico, se calcula con la siguiente fórmula de Betz (Song y Dhinakaran, 2000):

$$
\mathrm{P}=0.29 \mathrm{D}^{2} V^{3}
$$

Donde:

$$
\begin{aligned}
& \mathrm{P}=\text { potencia expresada en vatios }(\mathrm{W}) \\
& \mathrm{D}=\text { diámetro del rotor en metros }(\mathrm{m}) \\
& \mathrm{V}=\text { velocidad del viento por segundo }(\mathrm{m} / \mathrm{s})
\end{aligned}
$$

Un generador eólico pocas veces tendrá un rendimiento superior al 50\%, para no sobreestimar la potencia máxima dividiremos la fórmula Betz al 50\%.

$$
\mathrm{P}=0.15 \mathrm{D}^{2} V^{3}
$$

En el caso de tener un generador eólico con un diámetro de rotor de 5 metros y si el viento sopla a $10 \mathrm{~m} / \mathrm{s}(=25$ $\mathrm{km} / \mathrm{h}$ ), la potencia del molino será de $3.75 \mathrm{KW}$.

$$
\mathrm{P}=0.15 * 25 * 10^{3} \quad \mathrm{P}=3.75 \mathrm{KW}
$$

En esta investigación con un diámetro de rotor de 6 metros, si el viento sopla a $4.45 \mathrm{~m} / \mathrm{s}(=16.02 \mathrm{~km} / \mathrm{h})$, la potencia del molino será de $0.5 \mathrm{KW}$.

$$
\mathrm{P}=0.15 * 36^{*} 4.45^{3} \quad \mathrm{P}=0.46 \mathrm{KW}
$$

Para duplicar o triplicar la velocidad del generador, analizado en esta investigación, se utilizará un sistema mecánico, que garantice una velocidad mínima de $10 \mathrm{~m} / \mathrm{s}$. Es decir, si sopla a $10 \mathrm{~m} / \mathrm{s}$ la potencia será de $5.4 \mathrm{KW}$.

$$
\mathrm{P}=0.15 * 36 * 10^{3} \quad \mathrm{P}=5.4 \mathrm{KW}
$$

Según el resultado obtenido, con los $5.4 \mathrm{KW}$ que generaría el equipo eólico, se podría garantizar el alumbrado de cuatro aulas o laboratorios de la Facultad de Ingeniería y Arquitectura, ya que aproximadamente se necesita $1 \mathrm{KW}$ por cada uno de ellos.

\subsection{Sistema de transmisión}

La energía mecánica que se obtiene en el rotor del generador ha de ser transmitida mediante un "Multiplicador de engranajes" con el cual conseguimos obtener del generador aquella velocidad de giro que nos interesa.

Normalmente, cuando hablamos de aerogeneradores es necesario, primero aumentar la velocidad de rotación de giro, debido a que normalmente los rotores tienen una velocidad de rotación muy baja.

El sistema posee un eje primario acoplado directamente al eje de rotación del captador, que mediante un multiplicador transmite la potencia al eje del generador (eje secundario).

La velocidad del viento produce la potencia de la rotación del rotor, a causa de sus bajas revoluciones de funcionamiento demanda que necesitemos un multiplicador de la velocidad. El multiplicador está calculado, especialmente, para el motor asíncrono que utilizamos.

El multiplicador estará ubicado por encima de la torre y protegido de las inclemencias del tiempo a fin de que su desgaste sea lo mínimo posible. El generador también será protegido.

\section{Conclusiones}

El uso de la energía eólica apoyará tanto en la reducción de los altos costos de energía eléctrica en la Facultad de Ingeniería y Arquitectura, como también será de apoyo durante un corte de energía, de manera que se tenga disponible una fuente energética que permita cubrir las necesidades críticas en ese momento.

En una segunda parte de esta investigación, se pretende evaluar otras fuentes de energía renovables como es la energía solar y compararla con la energía eólica, a fin de determi- 
nar cuál de estos generadores es el más conveniente para ser usado y que nos garantice un menor mantenimiento y por consiguiente, un menor costo.

\section{Referencias}

Al-Shemmeri, T. (2010). Wind Turbines. Denmark: Ventus Publishing.

Enríquez, G. (2009). Tecnologías de generación de energía eléctrica. México: Limusa.

Huler, S. (2004). Defining the Wind. NY: Crown Publishing Group.

Ibrahim, H., Ilinca, A. y Perron, J. (2008). Energy Storage Systems. Characteristics and Comparisons. Renewable and Sustainable Energy Reviews, 12, 12211250.

Nemes, C. y Munteanu, F. (2010). A probabilistic approach of the wind energy system performance. Proceedings of the 12th WSEAS International conference on Mathematical methods and computational techniques in electrical engineering, Rumania.

Nemes, C. y Munteanu, F. (2011). Green Ware: greening cloud-scale data centers to maximize the use of renewable energy. Proceding of the 12th International Middleware Conference, Lisboa, Portugal.

Qian, X. y He, Y. (2011). Wind Power Turbine and Its Aerodynamic Characteristics. Proceedings of the Third International Workshop on Education Technology and Computer Science.

Sanz-Casado, E, Garcia-Zorita, J., Eleazar, A., Larsen, B. e Ingwersen, P. (2007).
Variable selection for wind power prediction using particle swarm optimization. Proceedings of the 9th annual Conference on Genetic and evolutionary computation, London.

Sanz-Casado, E, Garcia-Zorita, J., Eleazar, A., Larsen, B. e Ingwersen, P. (2013). Renewable energy research 19952009 - a case study of wind power research in EU, Spain, Germany and Denmark. Journal Scientometrics, 95, 197-224.

San Martín, J., Zamora, I., Aperribay, V. y Eguía, P. (2010). Hybrid fuel cells technologies for electrical microgrids. Electric Power Systems Research, 80, 993-1005.

Silva, J. y Montoya, Z. (2008). Evaluación de la contaminación atmosférica en la zona metropolitana de Lima-Callao. Boletín Hidrometereológico del Perú, 15-19.

Song, Y., Dhinakaran, B. (2000). Variable speed control of wind turbines using nonlinear and adaptative algorithms. Journal of Wind Engineering and Industrial Aerodynamics, 85, 293-308.

Villarrubia, M. (2012). Ingeniería de la Energía Eólica ( $1^{\circ}$ ed.). España: Marcombo.

World Wind Energy Association (2013). Half-year Report (Informe). Germany: Autor. 